\title{
Inhibition of nitric oxide production of activated mice peritoneal macrophages is independent of the Toxoplasma gondii strain
}

\author{
João Cláudio Damasceno-Sá1, Fernanda Silva de Souza', Thiago Alves Teixeira dos Santos ${ }^{1,2}$, \\ Fábio Conceição de Oliveira ${ }^{1}$, Maria de Fátima Sarro da Silva', Raul Ramos Furtado Dias', \\ Wanderley de Souza ${ }^{3}$, Andrea Cristina Veto Arnholdt ${ }^{4}$, Sergio Henrique Seabra ${ }^{1,2}$, \\ Renato Augusto DaMatta ${ }^{1 /+}$
}

${ }^{1}$ Universidade Estadual do Norte Fluminense, Centro de Biociências e Biotecnologia, Laboratório de Biologia Celular e Tecidual, Campos dos Goytacazes, RJ, Brasil

${ }^{2}$ Centro Universitário Estadual da Zona Oeste, Colegiado de Ciências Biológicas e da Saúde, Laboratório de Tecnologia em Bioquímica e Microscopia, Rio de Janeiro, RJ, Brasil

${ }^{3}$ Universidade Federal do Rio de Janeiro, Instituto de Biofísica Carlos Chagas Filho, Laboratório de Ultraestrutura Celular Hertha Meyer, Rio de Janeiro, RJ, Brasil

${ }^{4}$ Universidade Estadual do Norte Fluminense, Centro de Biociências e Biotecnologia, Laboratório de Biologia do Reconhecer, Campos dos Goytacazes, RJ, Brasil

BACKGROUND Toxoplasma gondii causes toxoplasmosis and is controlled by activated macrophages. However, infection of macrophages by tachyzoites induces TGF- $\beta$ signaling (TGF-s) inhibiting nitric oxide (NO) production. NO inhibition may be a general escape mechanism of distinct $T$. gondii strains.

OBJECTIVES To evaluate in activated macrophages the capacity of $T$. gondii strains of different virulence and genetics (RH, type I; ME-49, type II; VEG, type III; P-Br, recombinant) to evade the NO microbicidal defense system and determine LC3 loading to the parasitophorous vacuole.

METHODS Activated peritoneal macrophages were infected with the different $T$. gondii strains, NO-production was evaluated by the Griess reagent, and inducible nitric oxide synthase expression, TGF-s, and LC3 localisation assayed by immunofluorescence.

FINDINGS Only RH persisted in macrophages, while VEG was more resistant than P-Br and ME-49. All strains induced TGF-s, degradation of inducible nitric oxide synthase, and NO-production inhibition from 2 to $24 \mathrm{~h}$ of infection, but only RH sustained these alterations for $48 \mathrm{~h}$. By $24 \mathrm{~h}$ of infection, TGF-s lowered in macrophages infected by ME-49, and P-Br, and NO-production recovered, while VEG sustained TGF-s and NO-production inhibition longer. LC3 loading to parasitophorous vacuole was strain-dependent: higher for ME-49, P-Br and VEG, lower for RH. All strains inhibited NO-production, but only RH sustained this effect probably because it persisted in macrophages due to additional evasive mechanisms as lower LC3 loading to parasitophorous vacuole.

MAIN CONCLUSIONS These results support that $T$. gondii can escape the NO microbicidal defense system at the initial phase of the infection, but only the virulent strain sustain this evasion mechanism.

Key words: activated macrophages - nitric oxide - inducible nitric oxide synthase - TGF- $\beta$ signaling - LC3 - virulence

Toxoplasma gondii is an intracellular parasite with wide world distribution in warmblood vertebrates causing toxoplasmosis. Most infections are latent and do not cause clinical manifestations. ${ }^{(1)}$ However, the parasite can kill immune-compromised individuals and cause fetal problems. ${ }^{(1)} T$. gondii strains isolated in North America and Europe are classically divided into three genetically clonal lineages: I, II, and III.(2) Type I strains are highly virulent in mice, while type II and type III are less. ${ }^{(3,4)}$ However, most isolates from South America are biologically and genetically different from those isolated

\section{doi: 10.1590/0074-02760200417}

Financial support: CNPq, FAPERJ, CAPES (Finance Code 001).

+ Corresponding author: renato@uenf.br

(D) https://orcid.org/0000-0002-0108-9268

Received 15 August 2020

Accepted 09 February 2021 from North America and Europe. ${ }^{(5)}$ These strains are known as "recombinant", with a few being characterised by molecular analysis and infection in mice. $\mathrm{P}-\mathrm{Br}$ is a strain classified as type I-III that shows low virulence and is cystogenic in mice. ${ }^{(6)}$

$T$. gondii infects the host cell by active invasion and forms a parasitophorous vacuole (PV) that does not fuse with endolysosomal compartments, protecting the parasite from host digestion. ${ }^{(7,8)}$ The absence of compartment fusion to the PV is related to proteins secreted by specialised organelles such as rhoptries. ${ }^{(9)}$ Some of these proteins, from the serine/threonine kinase family ${ }^{(10,11,12,13)}$ are incorporated in the PV membrane providing identity and avoiding fusion with the endolysosomal compartments. ${ }^{(14)}$ Rhoptry proteins, particularly ROP5 and ROP18 of type I T. gondii strains. ${ }^{(15,16)}$ are virulence factors that disarm the immunity-related GTPases (IRG) microbicidal system of mouse host cells activated by interferon-gamma (IFN- $\gamma){ }^{(12)}$ Strains of low virulence are destroyed in IFN- $\gamma$ activated mouse host cells by the action of IRG that associate to the PV causing vesicula- 
tion and rupture of its membrane. ${ }^{(13,17,18)}$ Recruitment of IRG to the PV depends on core autophagy proteins that decorate the PV with LC3 ${ }^{(19,20,21)}$ However, this LC3 decoration is not classical autophagy, it is a cellular function known as "autophagy-related processes". (22) LC3 is conjugated to phosphatidylethanolamine by ubiquitinlike enzymes of the autophagy pathway, loads to the PV, and is crucial for IRG to control $T$. gondii replication. ${ }^{(20,21,23)}$ These studies used only type II $T$. gondii strains, which are avirulent to mice, to analyse the involvement of LC3 in the loading of IRG to the PV and T. gondii growth control. Only one study compared types I, II, and III $T$. gondii strain in a human cell system activated with IFN- $\gamma$ showing that LC3, and other autophagy adaptor proteins, are loaded to the PV in a strain-specific manner, with type I strain better avoiding this decoration. ${ }^{(24)}$ Therefore, nothing is known about strain differences on the LC3 loading to the PV after infection of activated mouse peritoneal macrophages.

Macrophages activated with IFN- $\gamma$ express inducible nitric oxide (NO) synthase (iNOS) that produces NO, a microbicidal agent that controls $T$. gondii growth. $(25,26,27,28,29,30,31)$ However, $T$. gondii infection of macrophages inhibits the production of $\mathrm{NO}^{(27,28,29,30,31,32,33,34,35)}$ by degrading iNOS expression ${ }^{(27,28,30,31,33)}$ through the proteasomal pathway. ${ }^{(36)}$ We have recently demonstrated that depending on the macrophage lineage $T$. gondii infection does not involve iNOS degradation, but NO production inhibition is constant. ${ }^{(33)}$ Also, ROPs $(5,16,17$, $17-18,18)$ were not essential for NO production inhibition. ${ }^{(33)}$ TGF- $\beta$ signaling is involved in the inhibition of $\mathrm{NO}$ production. ${ }^{(31)} \mathrm{NO}$ is a microbicidal agent crucial in vertebrates including mammals and birds that are hosts for $T$. gondii. Therefore, most $T$. gondii strains may need to deal with this microbicidal system. However, nothing is known about the induction of TGF- $\beta$ signaling and avoidance of NO production of activated macrophages by distinct $T$. gondii strains. Hence, we investigated the persistence, NO inhibition, iNOS expression, TGF- $\beta$ signaling, and LC3 loading to the PV, using four strains of T. gondii of different genetic backgrounds (RH, ME-49, VEG, $\mathrm{P}-\mathrm{Br}$ ) after infection of activated mice peritoneal macrophages to determine if $\mathrm{NO}$ inhibition is a possible general immune evasion strategy for this parasite.

\section{MATERIALS AND METHODS}

Macrophages and activation - Macrophages from the peritoneal cavity of male Swiss mice (6 weeks) were seeded on glass coverslips in 24-well plates. After $1 \mathrm{~h}$ at $37^{\circ} \mathrm{C}$ in a $5 \% \mathrm{CO}_{2}$ atmosphere, cells were washed and cultured for $24 \mathrm{~h}$ in Dulbecco's modified Eagle's medium (DMEM) containing 10\% fetal bovine serum, 100 $\mathrm{U} / \mathrm{mL}$ recombinant mouse IFN- $\gamma$ (Sigma) and $0.1 \mu \mathrm{g} / \mathrm{mL}$ of Escherichia coli lipopolysaccharide (0111:B4, LPS, Sigma) to activate macrophages. ${ }^{(30)}$

Ethical statement - This study was carried out in strict accordance with the animal experimentation Brazilian Law \#11794/08. The protocol was reviewed and approved by the Committee on the Ethics of Animal Experiments of the Universidade Estadual do Norte
Fluminense (UENF) (Permit Number: 98). Mice were euthanised with $\mathrm{CO}_{2}$ by following the recommendations in the Guide for the Care and Use of Laboratory Animals of the National Institutes of Health (NIH).

Parasites and animals - Tachyzoites of the RH, ME49, VEG, and P-Br strains were maintained by serial passage in Vero cells. Highly infected Vero cells without free tachyzoites in the supernatant were washed twice, scraped with a rubber policeman, and mechanically ruptured by passage through a syringe with a $26 \mathrm{G}$ needle, and the supernatant was filtered ( $3 \mu \mathrm{m}$ - Millipore membrane). The supernatant, containing tachyzoites, was collected and further centrifuged at $1000 \mathrm{~g}$ for $10 \mathrm{~min}$ at $4^{\circ} \mathrm{C}$. Parasites were resuspended in DMEM.

In vitro interactions and quantification of parasitic infection and persistence - Activated macrophages were washed, and infected with a 10:1 T. gondii:macrophage ratio. After $2 \mathrm{~h}$, cells were washed and DMEM supplemented with foetal bovine serum (FBS), IFN- $\gamma$, and LPS were added. The cells were further cultivated for 24 and $48 \mathrm{~h}$. For quantification of the infection $(2 \mathrm{~h})$ and parasitic persistence ( 24 and $48 \mathrm{~h}$ ) macrophages were fixed in phosphate-buffered saline (PBS) containing 3\% formaldehyde, washed and stained with a $10 \%$ Giemsa solution, dehydrated in acetone-xylene solutions, mounted in Entellan, and observed with an optical Zeiss Axioplan microscope. Images were captured with an MRc5 AxioCam digital camera and processed with the Axiovision program. Percentage of infected macrophages, and the mean number of intracellular $T$. gondii per macrophage were quantified. 200 macrophages per coverslip were counted in triplicate for each experiment. ${ }^{(35)}$

Evaluation of NO production - The production of NO was assessed by the Griess reagent. Supernatants were combined 1:1 with the Griess reagent for $10 \mathrm{~min}$, the samples were analysed in a spectrophotometer (540 $\mathrm{nm}$ ), and NO production in $\mu \mathrm{M}$ was obtained by comparing readings with a sodium nitrite diluted in DMEM standard curve. ${ }^{(37)}$

Immunofluorescence of bradyzoite antigen, iNOS, phosphorylated Smad 2 and $L C 3 A$ - Infected macrophages over coverslips cultured for $2,6,12,24$, and $48 \mathrm{~h}$ were collected and fixed in PBS containing 3\% formaldehyde, washed and incubated for 10 min in PBS containing $0.5 \%$ Triton X-100. Cells were incubated with $100 \mathrm{mM}$ ammonium chloride in PBS and incubated for $10 \mathrm{~min}$ in PBS containing 3\% BSA (PBS-BSA). Cells were incubated for $1 \mathrm{~h}$ in the serum of mice chronically infected with $T$. gondii diluted 1:5000 in PBS-BSA and rabbit polyclonal antibodies that recognise BAG-1 ${ }^{(38)}$ (kindly provided by Dr Louis Weiss), iNOS (M 19, Santa Cruz Biotechnology), phosphorylated Smad2 (P-SMAD2) (\# 3101, Cell Signaling Technology) or LC3A (L8793, Sigma), all diluted 1:200 in PBS-bovine serum albumin (PBS-BSA). Cells were washed with PBS-BSA and incubated with secondary antibodies against mouse (Alexa 594) and rabbit (Alexa 488) antibodies, diluted 1:100 in PBS-BSA, washed with PBS, mounted in ProLong Gold containing advanced diagnostic imaging (DAPI), and observed 
TABLE

Nitric oxide production of mouse peritoneal macrophages activated with interferon- $\gamma$ and lipopolysaccharide, infected with tachyzoites of the RH, ME-49, P-Br or VEG strains of Toxoplasma gondii ${ }^{a}$

\begin{tabular}{lcccc}
\hline & \multicolumn{3}{c}{ Nitrite production $(\mu \mathrm{M})$} \\
\cline { 2 - 5 } Macrophages & $24 \mathrm{~h}$ & $\%$ inhibition $24 \mathrm{~h}$ & $48 \mathrm{~h}$ & $\%$ inhibition $48 \mathrm{~h}$ \\
\hline Uninfected & $31.6 \pm 1.62^{b}$ & - & $38.3 \pm 0.88$ & - \\
\hline$T$. gondii $\mathrm{RH}$ & $10.7 \pm 1.00^{c}$ & 66.1 & $19.4 \pm 0.20^{c}$ & 49.4 \\
\hline T. gondii $\mathrm{ME}-49$ & $21.3 \pm 0.61^{c, d, e}$ & 32.6 & $45.6 \pm 1.25^{c, d, e}$ & 0 \\
\hline T. gondii $\mathrm{P}-\mathrm{Br}$ & $20.1 \pm 5.75^{c, d, e}$ & 36.4 & $42.4 \pm 5.20^{d, e}$ & 0 \\
\hline T. gondii $\mathrm{VEG}$ & $14.7 \pm 2.14^{c}$ & 53.5 & $31.2 \pm 0.84^{c, d}$ & 18.6 \\
\hline
\end{tabular}

$a$ : macrophages were activated $24 \mathrm{~h}$ prior to infection with tachyzoites of the different strains of $T$. gondii. After $2 \mathrm{~h}$, cells were washed, further cultured for 24 and $48 \mathrm{~h}$ and nitrite evaluated in the supernatant; $b$ : values are means \pm standard deviation of four independent experiments; $c$ : significantly different $(\mathrm{p}<0.001)$ from respective values for uninfected macrophages as calculated by analysis of variance (ANOVA); $d$ : significantly different $(\mathrm{p}<0.001)$ from respective values for RH-infected macrophages as calculated by ANOVA; $e$ : significantly different $(\mathrm{p}<0.01)$ from respective values for VEG-infected macrophages as calculated by ANOVA.

in the Axioplan microscope equipped with an $\mathrm{HBO} 100$ mercury lamp. Images were captured as before and processed with Adobe Photoshop. At least 100 macrophages per coverslips were quantified discriminating nuclear positivity for P-SMAD2. At least 100 tachyzoites per coverslips associated or not to vesicle positive for LC3A were quantified. Both quantifications were performed in triplicates in three independent experiments and means and standard deviations were calculated.

Data analyses - Values are expressed as means and standard deviations and were analysed statistically using one-way and two-way analysis of variance (ANOVA) and Tukey-Kramer multiple comparisons test. Repetition of experiments and $p$ values are indicated in the legends of the figures and table.

\section{RESULTS}

Only the RH strain persisted in activated macrophage - Tachyzoites of the RH strain were found with preserved morphology in activated macrophages after 24 and $48 \mathrm{~h}$ of infection (Fig. 1A). However, the ME-49 and $\mathrm{P}-\mathrm{Br}$ strains exhibited altered morphology after $24 \mathrm{~h}$ and by $48 \mathrm{~h}$ intracellular structures resembling digested tachyzoites were observed (Fig. 1B). The VEG strain was found persisting in macrophages after 24 and $48 \mathrm{~h}$ (not shown), but RH was much easier to find. Quantification of the percentage of infected macrophages by $T$. gondii revealed that the RH strain presented the lowest entrance value in the macrophage population as verified after $2 \mathrm{~h}$ of interaction; the other three strains had similar entrance rates (Fig. 1C, F). The RH strain was able to persist in macrophages as seen by the maintenance of the percentage of infected macrophages as infection progressed (Fig. 1D-F). The persistence capacity of the VEG strain decreased after 24 and $48 \mathrm{~h}$ of infection but was higher compared to ME-49 and P-Br strains that almost disappeared from macrophages over the examined time points (Fig. 1D-F). The mean number of intracel- lular T. gondii for the RH strain tended to increase as infection progressed, but the other three strains decreased (not shown). Parasites of the RH strain were negative for Bag antigen after $24 \mathrm{~h}$ of infection (not shown), indicating their tachyzoite stage during persistence.

All strains inhibited the NO microbicidal defense system after infection, but only the RH strain sustained this effect - NO production was effectively inhibited by tachyzoites of the RH strain after 24 and $48 \mathrm{~h}$ of infection and to a lesser extent by the VEG strain (Table). ME-49 and P-Br strains were also capable of inhibiting NO production by macrophages at $24 \mathrm{~h}$, but with a twofold lower capacity compared to the RH strain (Table). After $48 \mathrm{~h}$ macrophages infected by ME-49 strain produced more NO compared to uninfected macrophages and $\mathrm{P}-\mathrm{Br}$ strain did not inhibit NO production (Table). The VEG strain also had a lower capacity to inhibit NO compared to the RH strain at $48 \mathrm{~h}$ (Table).

Tachyzoites of all tested strains decreased iNOS expression by activated macrophages after $2 \mathrm{~h}$ of infection (Fig. 2B-E) compared to uninfected macrophages (Fig. 2A, F, K, P). The presence of tachyzoites infecting macrophages caused the low expression of iNOS with clear contrast to uninfected neighboring cells (Fig. 2B-E). After $6 \mathrm{~h}$ of infection, a few parasites of the ME-49 strain were observed inside macrophages that had an intermediate expression of iNOS (Fig. $2 \mathrm{H}$ ), but $\mathrm{P}-\mathrm{Br}$ and VEG strains were able to inhibit iNOS expression (Fig. 2I, J), but this inhibition was not as extensive compared to $\mathrm{RH}$ strain (Fig. 2G). After $24 \mathrm{~h}$ of infection, macrophages infected by the RH strain continue not expressing iNOS (Fig. 2L); macrophages infected with ME-49 or P-Br strains that presented $T$. gondii structures (digested tachyzoites), recovered iNOS expression (Fig. 2M, N). VEG tachyzoites maintained the inhibition capacity of iNOS expression after $24 \mathrm{~h}$ of infection (Fig. 2O). After $48 \mathrm{~h}$, macrophages infected with the RH strain had tachyzoites with preserved morphology and inhibited 

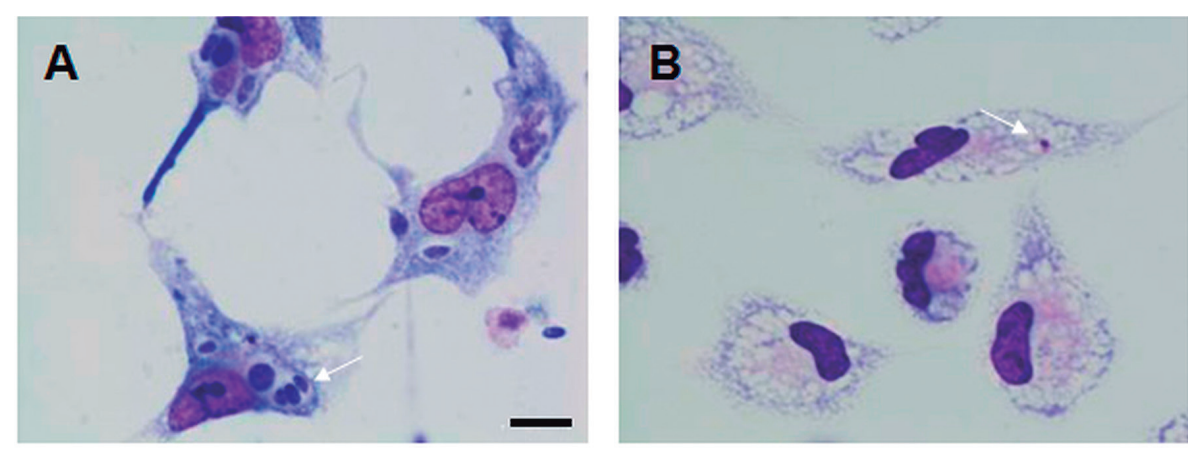

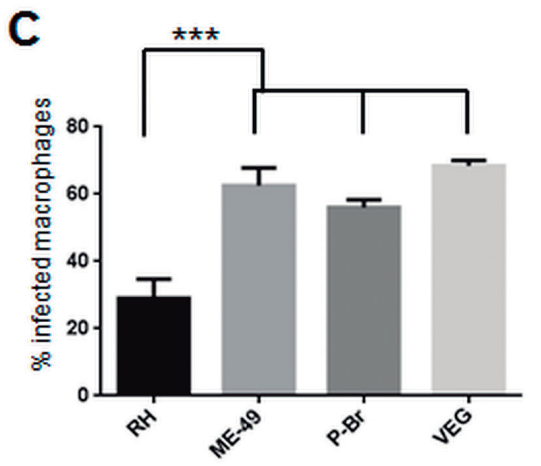

$2 \mathrm{~h}$

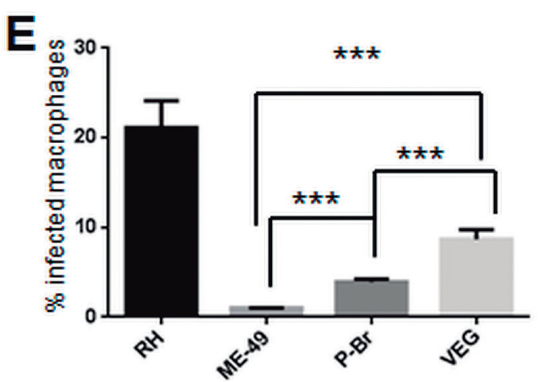

$48 \mathrm{~h}$

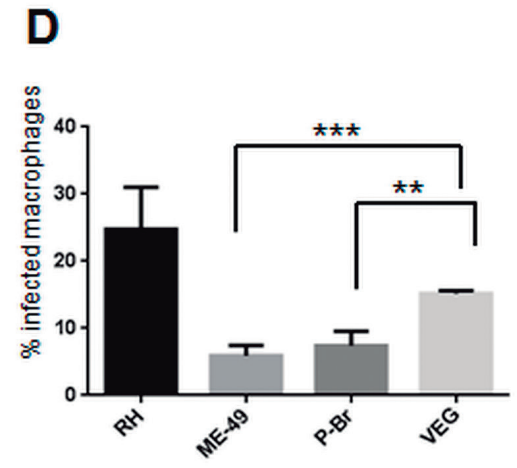

$24 \mathrm{~h}$

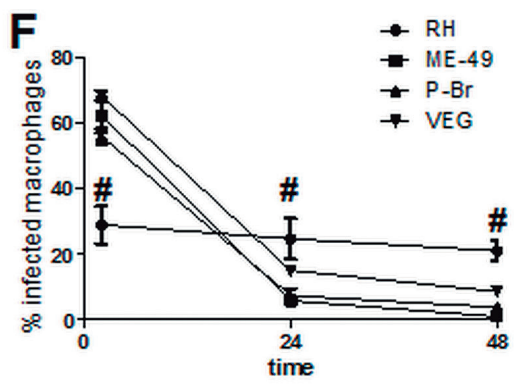

Fig. 1: bright-field images of Giemsa stained activated mouse macrophages infected for $48 \mathrm{~h}$ with Toxoplasma gondii (arrows) of the RH (A) and ME-49 (B) strains. Bar: $5 \mu \mathrm{m}$. Percentage of infected macrophages after 2 (C), 24 (D), and $48 \mathrm{~h}$ (E) of infection of activated macrophages. Results are expressed as means \pm standard deviations in triplicate of six independent experiments. A one-way analysis of variance (ANOVA) was performed, and a Tukey-Kramer multiple comparisons test was applied for comparison between strains $(\mathrm{C}, \mathrm{D}$, and $\mathrm{E})$. P values are noted as: ***p $<$ $0.001 ; * * \mathrm{p}<0.01$. A two-way ANOVA was performed $(\mathrm{F})$ to compare the persistence of infection between $\mathrm{RH}$ and the other strains, $\# \mathrm{p}<0.001$.

iNOS expression (Fig. 2Q). In contrast, macrophages infected with ME-49 and P-Br fully recovered iNOS expression and parasite remains were difficult to observe (Fig. 2R, S); macrophages infected with VEG recovered iNOS expression (Fig. 2T).

All tested strains induced TGF- $\beta$ signaling, sustained only by the RH strain - TGF- $\beta$ signaling involves the phosphorylation of Smad2 that is translocated to the nucleus. ${ }^{(39)}$ This signal inhibits NO production by macrophages ${ }^{(40)}$ and is involved in the capacity of $T$. gondii to inhibit the expression of iNOS. ${ }^{(31)}$ To determine if TGF- $\beta$ signaling was involved in the inhibition of iNOS expression after infection with the tested strains, nuclear translocation of P-SMAD2 during a $24 \mathrm{~h}$ infection period was verified.
Uninfected macrophages, independent of the observed time, had a low fluorescence signal for P-SMAD2 (Fig. $\left.3 \mathrm{~A}, \mathrm{~A}^{\prime}\right)$. After $2 \mathrm{~h}$ of infection with all tested strains, PSMAD2 was localised in the nucleus of infected macrophages (Fig. 3B, B'); the presence and persistence of P-SMAD2 in the nucleus of macrophages were quantified along with the infection between strains (Fig. 3C). Nuclear translocation of P-SMAD2 persisted for 6 and 24 $\mathrm{h}$ only in macrophages infected with the RH strain (Fig. 3C). When macrophages were infected with ME-49 or $\mathrm{P}-\mathrm{Br}$ strains P-SMAD2 nuclear translocation decreased as infection progressed (Fig. 3C) and infection with VEG strain caused an intermediate response in P-SMAD2 nuclear translocation between RH and ME-49 (Fig. 3C). 

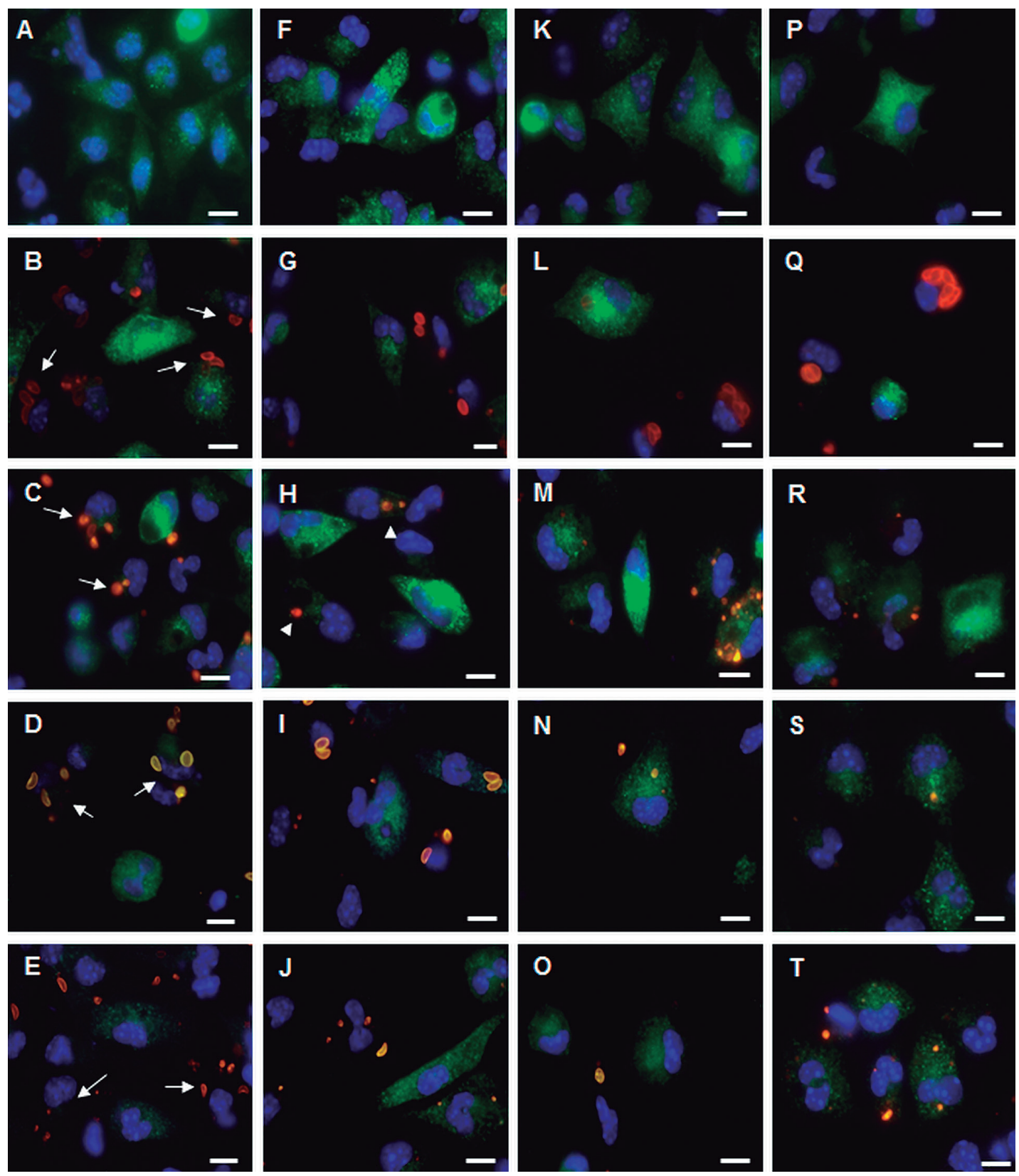

Fig. 2: immunofluorescence of nitric oxide synthase (iNOS) expression (green) and Toxoplasma gondii (red) in infected macrophages (blue nuclei). Macrophages after $2 \mathrm{~h}$ without infection (A) or after infection with strains RH (B), ME-49 (C), P-Br (D), or VEG (E). Infected macrophages (arrow) have reduced iNOS expression. Macrophages after $6 \mathrm{~h}$ without infection (F) or after infection with strains RH (G), ME-49 (H), $\mathrm{P}-\mathrm{Br}(\mathrm{I})$, or VEG (J). Macrophages infected with ME-49 (arrowhead) have an intermediate level of iNOS expression. Macrophages after $24 \mathrm{~h}$ without infection (K) or after infection with strains RH (L), ME-49 (M), P-Br (N), or VEG (O). Macrophages after 48 h without infection (P) or after infection with strains RH (Q), ME-49 (R), P-Br (S), or VEG (T). Bars: $10 \mu \mathrm{m}$. Representative images from four independent experiments.

RH was the only strain that avoided LC3 recruitment to the PV - Immunofluorescence microscopy showed that LC3 appeared as dots in the cytoplasm of uninfected activated macrophages (Fig. 4A). After $2 \mathrm{~h}$ of infection, LC3 was not easily associated with the PV of the different strains tested, particularly RH (Fig. 4B). However, as infection proceeded, LC3 associated more with the PV especially of the ME-49 (Fig. 4C) and P-Br strains. The association of LC3 with the PV were quantified and confirmed that the RH was the only strain of the ones used that maintained a low association of LC3 with the PV (Fig. 4D), that the ME-49 and PB-r strains had a higher association that increased with time and that the VEG strain presented an intermediate response (Fig. 4D).

\section{DISCUSSION}

T. gondii has many strategies to evade the host's immune system. One of the microbicidal systems this parasite needs to cope with is the production of NO by activated macrophages. We have demonstrated that 
tachyzoites of $T$. gondii of the RH strain can inhibit NO production of mice peritoneal macrophages by degrading iNOS through TGF- $\beta$ signaling. ${ }^{(31)}$ To verify if this is a general evasion mechanism, tachyzoites of four strains of $T$. gondii with different virulence were used to infect activated macrophages.
RH was the only strain that could persist in activated macrophages during the infection period analysed. The persistence of tachyzoites of the RH strain in activated macrophages has been reported before, ${ }^{(30,31)}$ and as far as we know, no comparison between strains of distinct virulence has ever been reported after infection of acti-
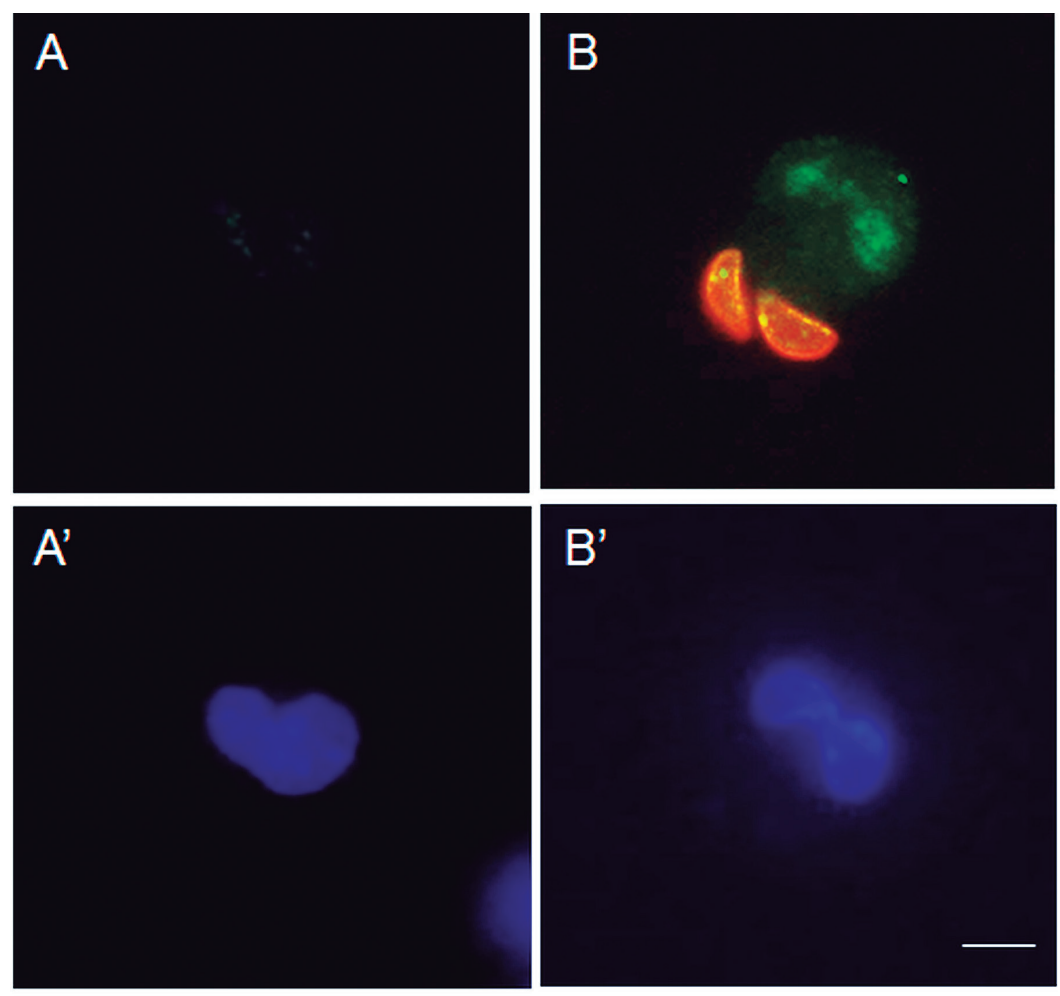

\section{C}

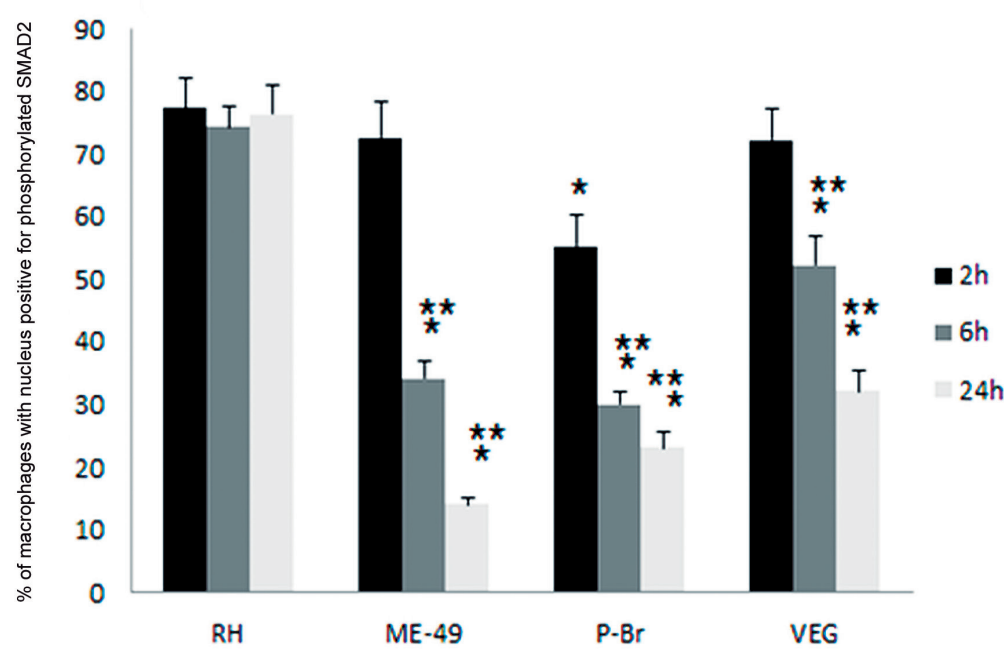

Fig. 3: immunofluorescence of Toxoplasma gondii (red), nuclear translocation of phosphorylated Smad2 (green) in infected macrophages (blue nuclei). Macrophages after $2 \mathrm{~h}$ without infection (A, A') or after infection with T. gondii (B, B'); a 2 h infection by the used strains caused similar nuclear translocation of phosphorylated Smad2. DAPI-stained nuclei of macrophages can be seen in the images with upper case letters with apostrophe (A', B'). Bars: $10 \mu \mathrm{m}$. Representative images from three independent experiments. (C) Quantification of macrophages with nucleus positive for phosphorylated Smad2 in percentage after 2, 6, and $24 \mathrm{~h}$ of infection with the RH, ME-49, P-Br, and VEG strains. Results are expressed as means \pm standard deviations in triplicate of three independent experiments. *Significantly different in relation to the value of $\mathrm{RH}$ after $2 \mathrm{~h}$ of infection by one-way analysis of variance (ANOVA) $(\mathrm{p}<0.05)$. $* *$ Significantly different in relation to the value of $2 \mathrm{~h}$ of infection within the same strain by one-way ANOVA $(\mathrm{p}<0.05)$. 
vated macrophages. RH was the only tested strain capable of persisting in activated macrophages, VEG strain persisted less but better than ME-49 and P-Br. The RH strain was quite suitable in resisting the microbicidal mechanisms of activated macrophages.

All strains could degrade iNOS right after macrophage infection $(2 \mathrm{~h})$. However, recovery of iNOS expression and the subsequent NO production of infected macrophages were strain-specific. ME- 49 and $\mathrm{P}-\mathrm{Br}$ were the strains with the least capacity to avoid the return of iNOS expression in macrophages. Macrophages infected with VEG strain also recovered iNOS expression and NO production, but at lower levels compared to ME-49. Only the RH strain sustained this inhibition. This indicates that all strains inhibited the NO microbicidal defense system after interacting with macrophage, but RH was the only strain that sustained this effect from inside the PV. The other strains did not sustain the inhibition of iNOS expression, probably because they were killed by other microbicidal systems (discussed below) allowing the macrophage to regain the capacity to respond to IFN- $\gamma$ and LPS present in the culture medium.

TGF- $\beta$ signaling was assayed to further understand the evasion mechanism of $T$. gondii that inhibits the NO microbicidal defense system. Only the RH strain main- tained TGF- $\beta$ signaling for longer periods; the VEG strain was capable of inducing longer TGF- $\beta$ signaling in comparison to ME-49 and P-Br strains that were capable of inducing it only during the initial infection. Thus, all strains were capable of inducing TGF- $\beta$ signaling soon after infection, but only RH maintained this signal long enough to inhibit iNOS expression for the entire period examined. The VEG strain sustained this signaling for a longer period compared to the ME-49 and P-Br strains, explaining its improved capacity to persist in macrophages.

Finally, we examined the loading of LC3 to the PV in macrophages. PV of activated mice peritoneal macrophages harboring parasites of the RH strain presented the least LC3 loading concerning the other strains, indicating a strain-dependent response in this host cell. It has been demonstrated that LC3 loads to the PV of IFN- $\gamma$ activated HeLa cells in a strain-specific manner with type I strain being able to better avoid this decoration than type II and III strains. ${ }^{(24)}$ Thus, it was not surprising that in activated macrophages LC3 loaded to the PV in a strain-specific way. LC3 loading to the PV is necessary for IRG accumulation onto the PV resulting in $T$. gondii growth arrest. ${ }^{(19,20,21)}$ IRGs control type II and III $T$. gondii strains, ${ }^{(12,15,23)}$ but type I has special ROPs $(5,17$, and 18) that phosphorylate IRGs, neutralising their microbicidal
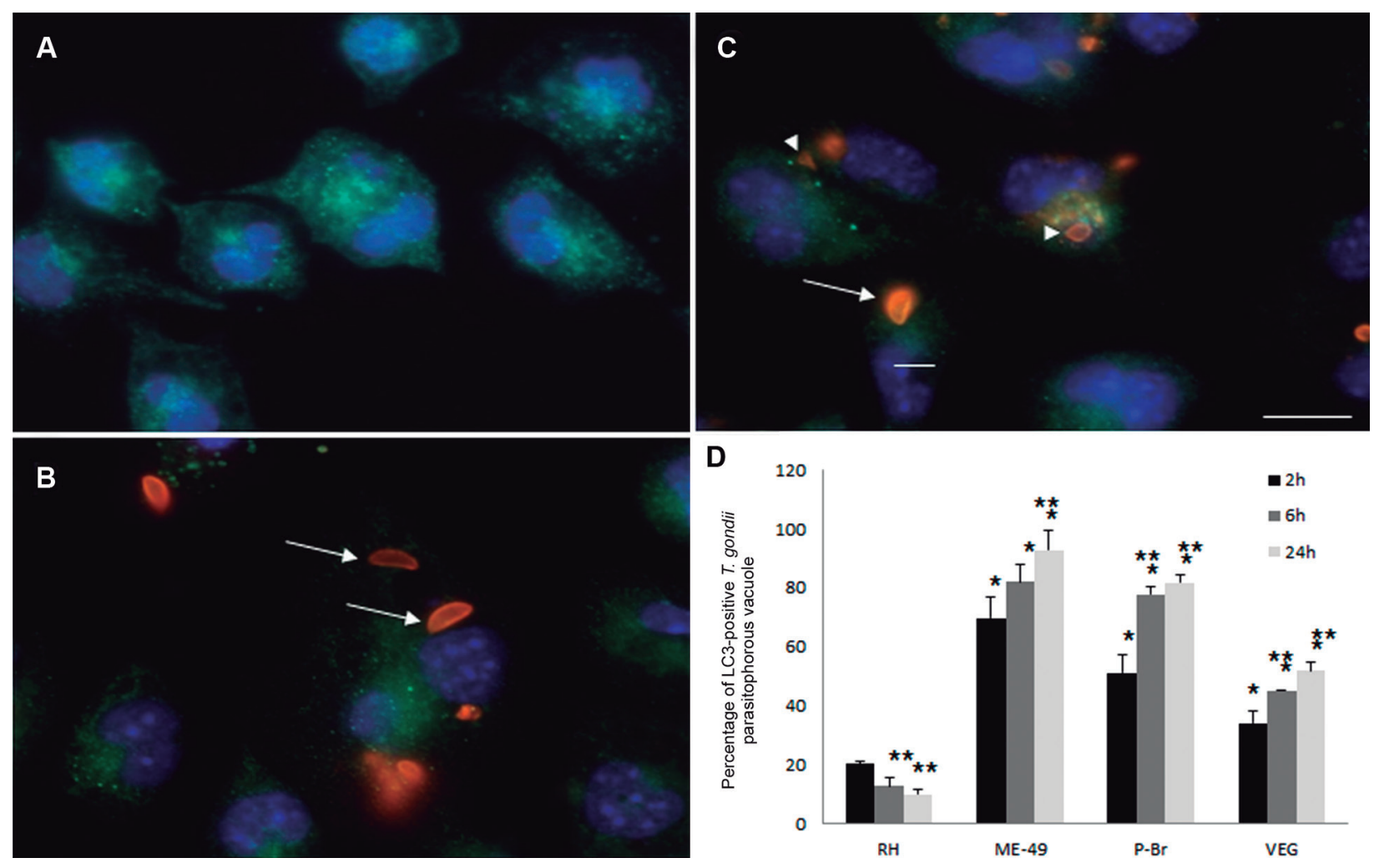

Fig. 4: immunofluorescence of LC3 (green) and Toxoplasma gondii (red) in infected macrophages (blue nuclei). Activated macrophages without infection (A) or after $2 \mathrm{~h}$ of infection with RH (B) or ME-49 (C) strains. Tachyzoites in parasitophorous vacuoles without (arrows) or associated (arrowheads) to LC3 (green) can be observed. Bars: $10 \mu \mathrm{m}$. Representative images from three independent experiments. Percentage of LC3-positive $T$. gondii parasitophorous vacuoles after 2, 6, and $24 \mathrm{~h}$ of infection with the RH, ME-49, P-Br, and VEG strains (D). Results are expressed as means \pm standard deviations in triplicate of three independent experiments. *Significantly different in relation to the value of $\mathrm{RH}$ after $2 \mathrm{~h}$ of infection by one-way analysis of variance (ANOVA) $(\mathrm{p}<0.05)$. **Significantly different in relation to the value of $2 \mathrm{~h}$ of infection within the same strain by one-way ANOVA $(\mathrm{p}<0.05)$. 
effect against the parasite. ${ }^{(11,13)}$ The persistence of type I T. gondii strains in IFN- $\gamma$ activated mice host cells indicates that these ROPs are virulence factors. ${ }^{(13)}$ Therefore, in addition to the capacity of type I strains to avoid IRGs by their unique ROPs, the capacity to impair LC3 loading to the PV adds to the evasion mechanisms of this type of strain, explaining its higher virulence in mice. This is further corroborated by the analysis of the influence of ROP 5,17 , and 18 on the capacity of $T$. gondii to inhibit NO production of activated mice macrophages. ${ }^{(33)}$ We have recently demonstrated that ROP 5, 17, and 18 are not essential for $\mathrm{NO}$ production inhibition after infection of activated mice macrophage. The lack of these ROPs in knockouts of the RH strain background was not essential for their persistence in activated macrophages; these mutants were not killed, sustaining macrophages in a nonactivated state as seen by low NO production. ${ }^{(33)}$ Thus, these RH knockouts were probably escaping death due to the lower LC3 loading, which reduces the association of IRGs to the PV. This results in better survival and maintenance of NO production inhibition. The capacity of the RH strain to avoid LC3 loading to the PV as described here probably increases parasite survival in these macrophages, resulting in persistence, the sustainability of the TGF- $\beta$ signaling, and NO production inhibition. On the other hand, ME-49 (type II), PB-r (recombinant), and VEG (type III) were capable of avoiding NO production during the initial hours of infection but probably did not sustain NO production inhibition because they could not avoid the IRG microbicidal system causing their death, return of macrophage response to IFN- $\gamma$ and LPS, and reexpression of iNOS and NO production. Collectively, the capacity of the RH strain of T. gondii to avoid LC3 loading to the PV in activated macrophages is probably an advantage over the other three strains used in this work, leading to higher persistence in activated macrophage.

All strains examined in this work were capable of inducing TGF- $\beta$ signaling that deactivates macrophages leading to iNOS degradation and inhibition of NO production. However, the virulent strain (RH), contrary to the other strains used, also could avoid LC3 loading to their PV. The evasive mechanism based on NO production inhibition may be present in $T$. gondii due to the presence of this microbicidal system in most hosts infected by this parasite. Thus, this inhibition capacity may be a general evasive strategy of all strains of $T$. gondii.

\section{ACKNOWLEDGEMENTS}

To Andrèa C César for reviewing the manuscript; Rosemary Cardoso Maciel for technical assistance; Dr Louis Weiss for the anti-bag antibody, and Ricardo Vitor, Lilian BahiaOliveira and Heitor Franco de Andrade Jr for kindly providing the P-Br, ME-49 and VEG strains, respectively.

\section{AUTHORS' CONTRIBUTION}

WS, ACVA, SHS and RAD conceived and designed the experiments; JCDS, FSS, TATS, FCO, MFSF and RRFD performed the experiments; JCDS, TATS, WS, ACVA, SHS and RAD analysed the data; RAD contributed reagents/materials/ analysis tools; JCDS and RAD authored the paper. The authors declare no conflict of interest.

\section{REFERENCES}

1. Hill DE, Chirukandoth S, Dubey JP. Biology and epidemiology of Toxoplasma gondii in man and animals. Anim Health Res Rev. 2005; 6(1): 41-61.

2. Howe DK, Sibley LD. Toxoplasma gondii comprises three clonal lineages: correlation of parasite genotype with human disease. J Infect Dis. 1995; 172(6): 1561-6.

3. Saeij JP, Boyle JP, Boothroyd JC. Differences among the three major strains of Toxoplasma gondii and their specific interactions with the infected host. Trends Parasitol. 2005; 21(10): 476-81.

4. Sibley LD, Boothroyd JC. Virulent strains of Toxoplasma gondii comprise a single clonal lineage. Nature. 1992; 359(6390): 82-5.

5. Vitaliano SN, Soares HS, Minervino AH, Santos AL, Werther K, Marvulo MF, et al. Genetic characterization of Toxoplasma gondii from Brazilian wildlife revealed abundant new genotypes. International journal for parasitology. Parasit Wildlife. 2014; 3(3): 276-83.

6. Ferreira AM, Vitor RW, Gazzinelli RT, Melo MN. Genetic analysis of natural recombinant Brazilian Toxoplasma gondii strains by multilocus PCR-RFLP. Infect Genet Evol. 2006; 6(1): 22-31.

7. Jones TC, Hirsch JG. The interaction between Toxoplasma gondii and mammalian cells. II. The absence of lysosomal fusion with phagocytic vacuoles containing living parasites. J Exp Med. 1972; 136(5): 1173-94.

8. Sinai AP, Joiner KA. Safe haven: the cell biology of nonfusogenic pathogen vacuoles. Annu Rev Microbiol. 1997; 51: 415-62.

9. Boothroyd JC, Dubremetz JF. Kiss and spit: the dual roles of Toxoplasma rhoptries. Nat Rev Microbiol. 2008; 6(1): 79-88.

10. Behnke MS, Khan A, Wootton JC, Dubey JP, Tang K, Sibley LD. Virulence differences in Toxoplasma mediated by amplification of a family of polymorphic pseudokinases. Proc Natl Acad Sci USA. 2011; 108(23): 9631-6.

11. Hakimi MA, Olias P, Sibley LD. Toxoplasma effectors targeting host signaling and transcription. Clin Microbiol Rev. 2017; 30(3): $615-45$.

12. Howard JC, Hunn JP, Steinfeldt T. The IRG protein-based resistance mechanism in mice and its relation to virulence in Toxoplasma gondii. Curr Opin Microbiol. 2011; 14(4): 414-21.

13. Hunter CA, Sibley LD. Modulation of innate immunity by Toxoplasma gondii virulence effectors. Nat Rev Microbiol. 2012; 10(11): 766-78.

14. Beckers CJ, Dubremetz JF, Mercereau-Puijalon O, Joiner KA. The Toxoplasma gondii rhoptry protein ROP 2 is inserted into the parasitophorous vacuole membrane, surrounding the intracellular parasite, and is exposed to the host cell cytoplasm. J Cell Biol. 1994; 127(4): 947-61.

15. Behnke MS, Fentress SJ, Mashayekhi M, Li LX, Taylor GA, Sibley LD. The polymorphic pseudokinase ROP5 controls virulence in Toxoplasma gondii by regulating the active kinase ROP18. PLoS Pathog. 2012; 8(11): e1002992.

16. Niedelman W, Gold DA, Rosowski EE, Sprokholt JK, Lim D, Farid Arenas A, et al. The rhoptry proteins ROP18 and ROP5 mediate Toxoplasma gondii evasion of the murine, but not the human, interferon-gamma response. PLoS Pathog. 2012; 8(6): e1002784.

17. Hunn JP, Feng CG, Sher A, Howard JC. The immunity-related GTPases in mammals: a fast-evolving cell-autonomous resistance system against intracellular pathogens. Mamm Genome. 2011; 22(1-2): 43-54.

18. Zhao Y, Ferguson DJ, Wilson DC, Howard JC, Sibley LD, Yap GS. Virulent Toxoplasma gondii evade immunity-related GTPasemediated parasite vacuole disruption within primed macrophages. J Immunol. 2009; 182(6): 3775-81. 
19. Besteiro $\mathrm{S}$. The role of host autophagy machinery in controlling Toxoplasma infection. Virulence. 2019; 10(1): 438-47.

20. Choi J, Park S, Biering SB, Selleck E, Liu CY, Zhang X, et al. The parasitophorous vacuole membrane of Toxoplasma gondii is targeted for disruption by ubiquitin-like conjugation systems of autophagy. Immunity. 2014; 40(6): 924-35.

21. Park S, Choi J, Biering SB, Dominici E, Williams LE, Hwang S. Targeting by AutophaGy proteins (TAG): Targeting of IFNG-inducible GTPases to membranes by the LC 3 conjugation system of autophagy. Autophagy. 2016; 12(7): 1153-67.

22. Subramani S, Malhotra V. Non-autophagic roles of autophagyrelated proteins. EMBO Rep. 2013; 14(2): 143-51.

23. Zhao Z, Fux B, Goodwin M, Dunay IR, Strong D, Miller BC, et al. Autophagosome-independent essential function for the autophagy protein Atg5 in cellular immunity to intracellular pathogens. Cell Host Microbe. 2008; 4(5): 458-69.

24. Selleck EM, Orchard RC, Lassen KG, Beatty WL, Xavier RJ, Levine B, et al. A noncanonical autophagy pathway restricts Toxoplasma gondii growth in a strain-specific manner in IFN-gammaactivated human cells. MBio. 2015; 6(5): e01157-15.

25. Adams LB, Hibbs Jr JB, Taintor RR, Krahenbuhl JL. Microbiostatic effect of murine-activated macrophages for Toxoplasma gondii. Role for synthesis of inorganic nitrogen oxides from Larginine. J Immunol. 1990; 144(7): 2725-9.

26. Gazzinelli RT, Oswald IP, James SL, Sher A. IL-10 inhibits parasite killing and nitrogen oxide production by IFN-gamma-activated macrophages. J Immunol. 1992; 148(6): 1792-6.

27. Guillermo LV, DaMatta RA. Nitric oxide inhibition after Toxoplasma gondii infection of chicken macrophage cell lines. Poult Sci. 2004; 83(5): 776-82

28. Luder CG, Algner M, Lang C, Bleicher N, Gross U. Reduced expression of the inducible nitric oxide synthase after infection with Toxoplasma gondii facilitates parasite replication in activated murine macrophages. Int J Parasitol. 2003; 33(8): 833-44.

29. Miranda FJ, Damasceno-Sa JC, DaMatta RA. Development of ostrich thrombocytes and monocyte-derived macrophages in culture and the control of Toxoplasma gondii reproduction after macrophage activation. Poult Sci. 2016; 95(1): 12-8.
30. Seabra SH, de Souza W, DaMatta RA. Toxoplasma gondii partially inhibits nitric oxide production of activated murine macrophages. Exp Parasitol. 2002; 100(1): 62-70.

31. Seabra SH, de Souza W, DaMatta RA. Toxoplasma gondii exposes phosphatidylserine inducing a TGF-betal autocrine effect orchestrating macrophage evasion. Biochem Biophys Res Commun. 2004; 324(2): 744-52.

32. Seabra SH, DaMatta RA, de Mello FG, de Souza W. Endogenous polyamine levels in macrophages is sufficient to support growth of Toxoplasma gondii. J Parasitol. 2004; 90(3): 455-60.

33. Cabral GRA, Wang ZT, Sibley LD, DaMatta RA. Inhibition of nitric oxide production in activated macrophages caused by Toxoplasma gondii infection occurs by distinct mechanisms in different mouse macrophage cell lines. Front Microbiol. 2018; 9: 1936.

34. DaMatta RA, Seabra SH, Manhaes L, de Souza W. Nitric oxide is not involved in the killing of Trypanosoma cruzi by chicken macrophages. Parasitol Res. 2000; 86(3): 239-43.

35. Santos TA, Portes JA, Damasceno-Sá JC, Caldas LA, Souza W, DaMatta RA, et al. Phosphatidylserine exposure by Toxoplasma gondii is fundamental to balance the immune response granting survival of the parasite and of the host. PLoS One. 2011; 6(11): e27867.

36. Padrao JC, Cabral GR, da Silva MF, Seabra SH, DaMatta RA. Toxoplasma gondii infection of activated J774-A1 macrophages causes inducible nitric oxide synthase degradation by the proteasome pathway. Parasitol Int. 2014; 63(5): 659-63.

37. Green LC, Wagner DA, Glogowski J, Skipper PL, Wishnok JS, Tannenbaum SR. Analysis of nitrate, nitrite, and $[15 \mathrm{~N}]$ nitrate in biological fluids. Anal Biochem. 1982; 126(1): 131-8.

38. Weiss LM, LaPlace D, Tanowitz HB, Wittner M. Identification of Toxoplasma gondii bradyzoite-specific monoclonal antibodies. J Infect Dis. 1992; 166(1): 213-5.

39. Massague J, Xi Q. TGF-beta control of stem cell differentiation genes. FEBS Lett. 2012; 586(14): 1953-8.

40. Freire-de-Lima CG, Xiao YQ, Gardai SJ, Bratton DL, Schiemann WP, Henson PM. Apoptotic cells, through transforming growth factor-beta, coordinately induce anti-inflammatory and suppress pro-inflammatory eicosanoid and $\mathrm{NO}$ synthesis in murine macrophages. J Biol Chem. 2006; 281(50): 38376-84. 